\title{
FEA analysis during extrusion of polygonal sections from round billet through linearly converging dies using SERR Technique
}

\author{
A. K. Rout $\dot{A}^{*}, \quad$ R. Das ${ }^{\dot{A}}$ and B. C. Routara $\dot{A}$ \\ ${ }^{\dot{A}}$ School of Mechanical Engineering, KIIT University, Bhubaneswar - 24, Odisha, India
}

Accepted 10 January 2014, Available online 01 February 2014, Special Issue-2, (February 2014)

\begin{abstract}
The extrusion of section from round billet poses a great challenge for theoretical modeling of the process using upper bound method. The greatest difficulty in three-dimensional upper bound method is to determine kinematically admissible velocity field. The SERR (Spatial Elementary Rigid Region) technique is fairly applicable for analyzing extrusion of sections having re-entrant corners. A modified version of SERR technique has been used for extrusion of hexagon sections from round billet through a linearly converging die. The circular cross section of the round billet is approximated by a regular polygon of equal area. The extrusion pressure has been computed for different boundary condition at the die billet interface. The optimum die geometry has been determined. An extrusion test rig is designed and all extrusion is carried out using round billets for extrusion of hexagonal sections using converging dies. The experimental results are validated with SERR analysis.
\end{abstract}

Keywords: SERR, Round, Hexagon, Extrusion, Converging Die.

\section{Introduction}

A number of analytical studies have been carried out during the past few years to compute the deformation load for extrusion/drawing of sections from round billets through square dies using three-dimensional (3D) upper bound theorem. The greatest difficulty in upper bound method is to obtain a kinematically admissible velocity field. SERR (Spatial elementary Rigid Region) (Gatto and Giardo, 1981) technique is applicable in the above cases. A number of investigators have applied the SERR technique to determine extrusion pressure in flat faced square dies. (Kar and Das, 1997) carried out upper bound analysis of extrusion of I-section from square/rectangular billets through flat faced square dies. (Sahoo et al., 1998) investigated the extrusion of channel section from square or rectangular billets. (Kar et al., 1977) carried out investigation of extrusion of square section from round billet using SERR technique for flat faced die and obtained extrusion pressure for different reductions. The flat faced die has the disadvantage of high extrusion load with high strain hardening characteristics. The converging dies are preferred to flat faced square dies as they render the deformation more homogeneous with consequent reduction in the deformation load. Despite the advantages of converging dies, only a few theoretical approaches to extrusion or drawing processes have been reported. (Gunasekera and Hoshino, 1982) investigated the extrusion and drawing of square sections from round billets through converging dies formed by an envelope of

*Corresponding author: A. K. Rout

DOI: http://dx.doi.org/10.14741/ijcet/spl.2.2014.111 straight lines using upper bound theorem. (S. K. Sahoo et al, 1999) obtained an upper-bound solution for the extrusion of hexagon section from round billet using a linearly converging die using SERR technique. It seems that adequate investigations have not been carried out for the extrusions of section from round billet using linearly converging die. In view of the advantage of linearly converging die a great deal of investigations are yet to be carried out to standardize the process parameter and to optimize the die design for extrusion of different sections. In the present investigation a reformulated SERR technique has been used for extrusion of hexagon section from round billet for rigid plastic material. The round billet has been approximated by regular polygon. The extrusion pressure has been determined for different reductions and optimum geometry. The solution is validated by experiments. It is observed that theory agrees well with the experiment.

\section{The SERR Technique}

In the SERR technique, the deformation zone is envisaged to consist of tetrahedral rigid blocks, each block separated from others by planes of velocity discontinuity. Each rigid region has its own internal velocity vector consistent with the boundary conditions. Thus, if there are $\mathrm{N}$ rigid blocks, then the number of unknown internal velocity vectors is also $\mathrm{N}$ (thus, $3 \mathrm{~N}$ spatial velocity components). The velocity at entry to the deformation zone (the billet velocity) is considered to be prescribed and the velocity at the exit has a single component since its direction is known from the physical description of the problem. 
Therefore the total number of unknown velocity components in the global level becomes $3 \mathrm{~N}+1$. All these unknown velocity components can be uniquely determined if an equal number of equations are generated. This is done applying the mass continuity condition to the bounding faces of all the tetrahedral rigid blocks taken together. It may be noted that the set of velocity equations so generated becomes consistent and determinate if and only if the SERR blocks are tetrahedral in shape, so that, the number of triangular bounding faces automatically becomes $3 \mathrm{~N}+1$.

\subsection{Discretisation of the Deformation Zone}

The deformation zone in case of metal forming that occurs in a closed channel (such as extrusion or drawing), can be sub-divided into sub-zones that are prismatic, pyramidal or tetrahedral in shape or a combination of these shapes. Since the elementary blocks are to be tetrahedral in nature, the prismatic or pyramidal sub-zones are discretised ultimately into tetrahedrons pyramid can be discretised into two tetrahedrons by dividing the quadrilateral base into two Triangles. Thus, there are two ways of discretising the pyramid into two tetrahedral blocks. The details of the discretisation are shown in Table-1.

Table 1 Discretisation details

\begin{tabular}{|l|l|}
\hline Type of sub-zones & 2 pyramids; 2 tetrahedrons \\
\hline $\begin{array}{l}\text { Total number of SERR } \\
\text { blocks }\end{array}$ & $2+2+1+1=6$ \\
\hline $\begin{array}{l}\text { Number of discretisation } \\
\text { schemes }\end{array}$ & $2 \times 2=4$ \\
\hline $\begin{array}{l}\text { Number of triangular } \\
\text { faces }\end{array}$ & 19 \\
\hline $\begin{array}{l}\text { No. of velocity } \\
\text { components }\end{array}$ & $\begin{array}{l}6 \times 3=19 \text { for } 6 \text { SERR and } 1 \text { at } \\
\text { exit; Total=19 }\end{array}$ \\
\hline
\end{tabular}

\subsection{Application of the upper-bound theorem}

The upper-bound theorem predicts the power necessary to perform the desired metal forming at the prescribed velocities. However, since the velocity field for a given problem is generally not known, the power for any velocity field as calculated by the upper-bound theorem is greater than or equal to the actual power. The development also applies to any velocity field which satisfies the boundary and plasticity requirements. Thus for any possible velocity field there also exists an associated total power. The actual velocity field is that which minimizes the associated total power and the actual total power is the minimum associated total power. The formal statement of the upper-bound theorem is that amongst all kinematically admissible velocity fields, the actual field minimizes the work-function $J$, where:

$J=J_{1}+J_{2}+J_{3}$

In which $\mathrm{J}_{1}$ is the work dissipated for internal deformation.

$J_{1}=\frac{2 \sigma_{0}}{\sqrt{3}} \int_{v} \sqrt{\frac{1}{2} \dot{\varepsilon}_{i j} \dot{\varepsilon}_{i j}} d v$
$\mathbf{J}_{2}$ is the work dissipated at surfaces of velocity discontinuity.

$$
J_{2}=\frac{\sigma_{0}}{\sqrt{3}} \int_{A_{i}}\left|\Delta v_{i}\right| d A_{i}
$$

And $J_{3}$ is the work dissipated due to friction at the diework piece interface (jth face):

$$
J_{3}=\frac{m \sigma_{0}}{\sqrt{3}} \int_{A f j}\left|\Delta v_{j}\right| d A_{f j}
$$

In the present formulation with a discontinuous velocity field the strain rate components $\varepsilon_{i j}$ are all zero inside the rigid blocks, This leads to: $\mathrm{J}_{1}=0$. Since, velocity discontinuities $\quad\left|\Delta V_{i}\right|$ and $\left|\Delta V_{j}\right|$ are constant over all of the faces:

$J=\frac{\sigma_{0}}{\sqrt{3}} \int\left|\Delta V_{i}\right| A_{i}+\left(\frac{m \sigma_{0}}{\sqrt{3}}\right) \int\left|\Delta V_{j}\right| A_{f j}$

The mean non-dimensional average extrusion pressure is determined from the relation:

$$
\frac{p_{a v}}{\sigma_{0}}=\frac{J}{W^{2} V_{b} \sigma_{0}}
$$

Where $p_{a v}$ is the average extrusion pressure and $\sigma_{0}$ is the flow stress

\section{Experiment}

An experimental set up was fabricated for extrusion of hexagonal section, which consists of container, die holder, punch and die sets. Before starting the tests, the die sets, the die holder and the inside faces of the extrusion chamber were cleaned with kerosene and then carbon tetrachloride. The round-to-hexagon converging die block was then push-fitted into the die holder and the assembly was secured by screwing the four allen screws with the container of the die set. The full assembly was then placed on the lower table of the universal testing machine.

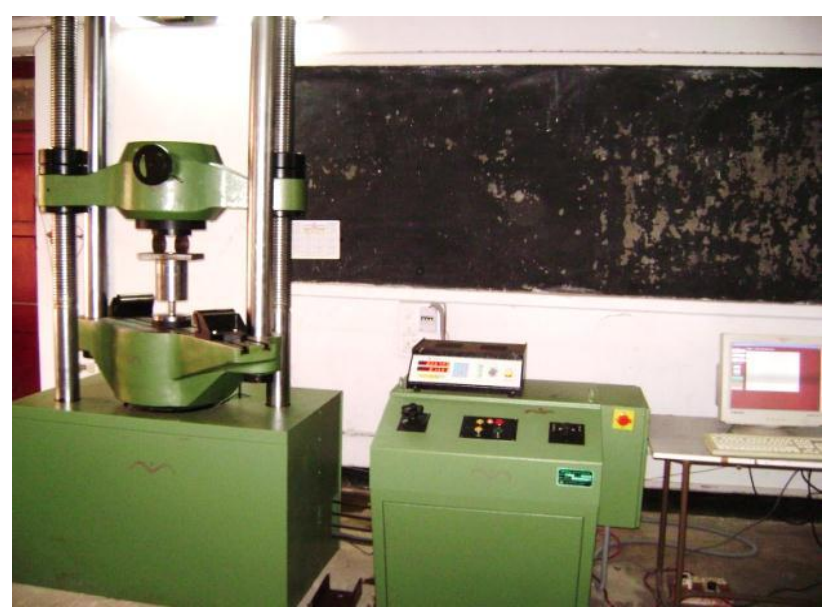

Fig.1. the experimental set-up mounted on UTM

For carrying out an extrusion test, the die block was kept in wet condition and the specimen was placed inside the extrusion chamber. The punch was then inserted into its position. After centering the apparatus on the machine lower table as shown in Fig.1, the machine was started and the extrusion process was continued. The lead 
(Composition: $\mathrm{Pb}-75 \%$, $\mathrm{Ca}-10 \%$ and $\mathrm{SiO}_{2}-15 \%$ ) was taken as the work material. Punch load was recorded at regular time interval of the punch travel by using personal computer. The application of load was continued until it started to rise again after reaching the steady state and the test was terminated. The die holder was then separated from the extrusion chamber and finally the die blocks with the extruded product were pushed out from the die holder. Experiments were conducted for three different reductions at wet conditions. The photographs of the extruded products as shown in Fig.2.

\section{Results and Discussion}

Computations were carried out for all four schemes of hexagonal section and the scheme giving the least upperbound was identified using a multivariable optimization technique (Kuester and Mize, 1973). The discretised deformation zone corresponding to the least upper-bound is named here as the optimum configuration. This optimum configuration is utilized for computation of the variation of the normalized extrusion pressure with equivalent semi-cone angle (in degrees) and the percentage area reduction, for different friction factors (Fig.3). It is clear from these figures that the optimal semicone angle that secures the minimal extrusion pressure increases with the increase of friction. The extrusion pressure increases with increase in reduction and friction factor. It is interesting to note that rise in extrusion pressure is more significant at high reduction than that of low reduction. These results can be used to predict the forming stress and optimal die shape for designing the sectioned die, assessing the frictional condition either in an empirical way or by means of a simulation test.

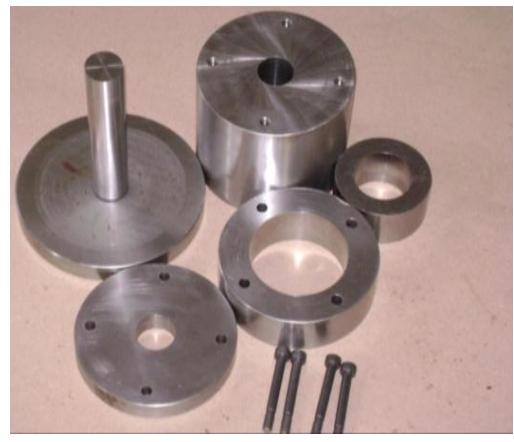

Fig.2. Photographs showing all parts of the set-up

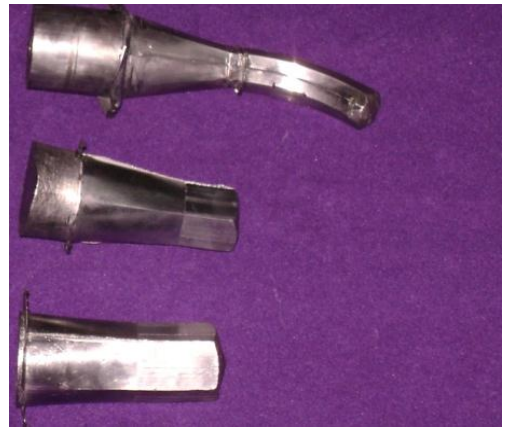

Fig.2. Extruded sections of different reduction
The present modeling is validated with experiments for wet friction condition. The extrusion was carried with lead specimens. The wet friction condition at the die-billet interface corresponds to constant friction factor $\mathrm{m}=0.40$ which was determined by ring compression test. The flow stress of the work material determined using uni-axial compression test and found to be $37.5 \mathrm{~N} / \mathrm{mm}^{2}$. The detail calculation of non-dimensional extrusion pressure is shown in Table-2.The variation of extrusion loads with ram travel as obtained from the experiments for $40 \%, 60 \%$ and $80 \%$ reductions are shown in Fig.4. It is evident that extrusion load increases with increase in reduction. It is obvious that the non-dimensional extrusion pressure obtained from experimental investigation agrees with the theoretically predicted value.

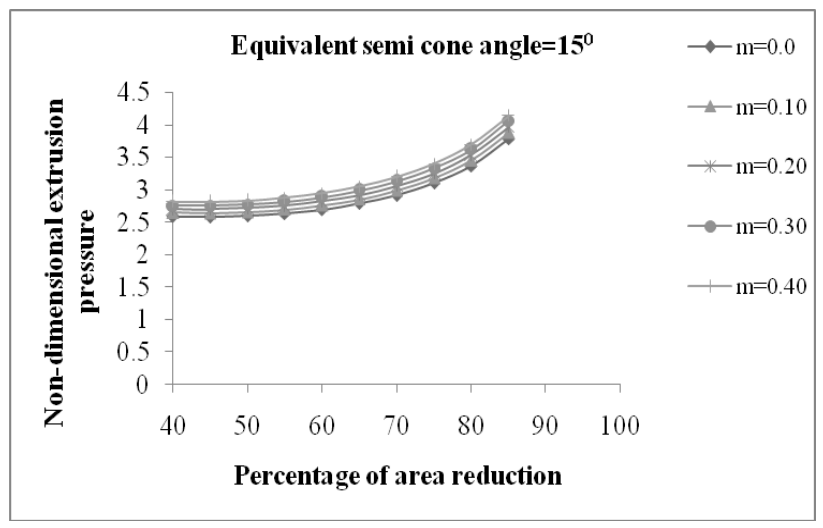

Fig.3. Effect of friction on the extrusion pressure

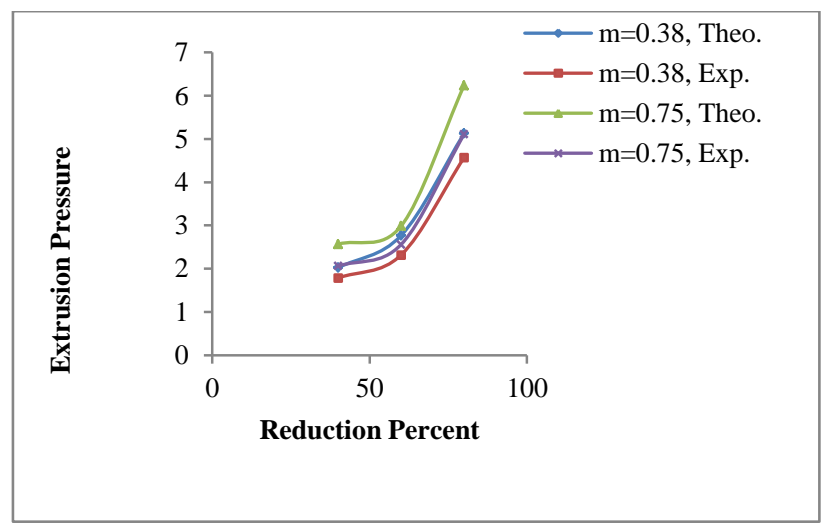

Fig.4. Extrusion pressure vs. Percent reduction

Table2 Comparison of experimental and computed results

\begin{tabular}{|l|l|l|l|l|l|}
\hline \multirow{2}{*}{$\begin{array}{l}\text { Reducti } \\
\text { on }(\%)\end{array}$} & Punch Load & \multirow{2}{*}{$\begin{array}{l}\mathrm{P}_{\mathrm{av}} \\
(\mathrm{N})\end{array}$} & $\sigma_{0}$ & $\mathrm{P}_{\mathrm{av}} / \sigma_{0}$ & \\
\cline { 5 - 7 }$\left(\mathrm{N} / \mathrm{mm}^{2}\right)$ & & & & Computed \\
\hline 40 & $63.5 \times 10^{3}$ & 89.834 & 37.5 & 2.395 & 2.810 \\
\hline 60 & $81 \times 10^{3}$ & 114.59 & 37.5 & 3.055 & 3.154 \\
\hline 80 & $96 \times 10^{3}$ & 135.8 & 37.5 & 3.621 & 3.695 \\
\hline
\end{tabular}

\section{Conclusions}

From the present study the following conclusions are drawn; (i) Using the present investigation, the optimal die geometry (the equivalent semi-cone angle), that requires the minimal extrusion pressure can be obtained for 
different reductions of area and friction conditions; (ii) The optimum cone angle increases with increase in friction at the die billet interface; (iii) From the comparison made with the experiment, it is observed that the theory agrees well with the experimental results and the error is nearly $10-15 \%$ in comparison to experimental results Hence the present solution can predict reasonable upper bound extrusion pressure.

\section{References}

F. Gatto, A. Giardo, (1981), the characteristics of threedimensional analysis of plastic deformation according to the SERR method, International Journal of Mechanical Science, 23, 129-148.

J. S. Gunasekera, S. Hosino, (1982), Analysis of extrusion or drawing of polygonal sections through straightly conversing dies, J. Eng. Ind. Trans, ASME, 104, 38-45.

P. K. Kar, N. S. Das, (1997), Upper bound analysis of extrusion of I-section bars from square/rectangular billets through square dies, International Journal of Mechanical Science, 39(8), 925-934.
P. K. Kar, R. K. Sahoo, (1977), an application of the SERR technique to the analysis of extrusion of square sections from round billets, Journal of Institution of Engineers (India), 78, 151-154.

J. L. Kuester, J. H. Mize, (1973), Optimization Techniques

R. Narayanasamy, P. Srinivasan, R. Venkatesan, (2003), computer aided design and manufacture of streamlined extrusion dies, International Journal of Materials Processing Technology, 138, 262-264

R. Narayanasamy, R. Ponalagusamy, R., Venkatesan, P. Srinivasan, (2006), An Upper bound solution to extrusion of circular billet to circular shape through cosine dies, International Journal of Materials and Design, 27 (5), 411415

R. Narayanasamy, K. Baskaran, S. Arunachalam, D. M. Krishna, (2008), an experimental investigation on barreling of aluminium alloy billets during extrusion-forging using different lubricants, International Journal of Materials and Design, 29, 2076-2088

R. Narayanasamy, K. Baskaran, D. Muralikrishna, (2008), some studies on stresses and strains of aluminium alloy during extrusion-forging at room temperature, International Journal of Materials and Design, 29, 1623-1632 